\title{
Medical Error as a Challenge in Iran's Health System
}

\author{
Mohammad Khammarnia,, ${ }^{1,}$ and Fatemeh Setoodehzadeh ${ }^{2}$ \\ ${ }^{1}$ Health Promotion Research Center, Zahedan University of Medical Sciences, Zahedan, IR Iran \\ ${ }^{2}$ Department of Health Management and Economics, School of Public Health, Tehran University of Medical Sciences, Tehran, IR Iran \\ "Corresponding author: Mohammad Khammarnia, Health Promotion Research Center, Zahedan University of Medical Sciences, Zahedan, IR Iran. E-mail: \\ m_khammar1985@yahoo.com
}

Received 2016 June 05; Revised 2016 August 23; Accepted 2016 September 16.

Keywords: Medical Error, Challenge, Iran

1. Definition of Medical Error

Medical error (ME) is defined as any error in carrying out planned action or any incorrect action for achieving a purpose in the health care system. These errors may arise in process, performance, product or system (1). Medical errors (MEs) in delivery of healthcare are a major threat to patients' safety (2) and could occur in any healthcare organization e.g. hospital, health center, laboratory, clinic etc. Moreover, preventable MEs are a leading cause of medical injuries worldwide (1). Medical error is a challenge in every country (e.g. about 225000 patients die annually in the United State as a result of ME), and health systems suffer from human and economic expenditures (costs imposed on both health systems and patients due to these errors) (2). In addition, it is one of the main causes of increasing complaints in hospitals and prolongs the hospital stay and in some cases results in death $(2,3)$.

Although there are various categorizations of MEs, classification to human errors (such as medication, diagnosis, prescription, documentation, treatment and coordination), systemic errors (administrational or organizational and process) and technical errors is the most common MEs categorization (4).

\section{Reasons of Medical Error}

The causes of ME vary such as misidentification (5), stress, crowding in wards, heavy workload, burnout, lack of communication among healthcare professionals, medical knowledge deficiency and not writing the drug form and drug dose by physicians, which could be potentially harmful to patients (6-8). The lack of reporting MEs through medical staff is another cause of increasing ME in hospitals (4).

\section{Medical Error; a Challenge in Iran}

As mentioned above, ME is a challenge in every country especially in Eastern Mediterranean region such as Iran (about 4.4 million MEs occurred in countries in the Eastern Mediterranean region)(9). The high rate of ME in some hospitals in Iran confirms the mentioned challenge (2) and it is known as a challenge in all hospitals in Iran, especially in public hospitals $(4,6)$. Although there is an ME registration system in most hospitals, with both voluntary and mandatory approach, its prevalence is not clear. Nevertheless, a study indicated that the prevalence of medication errors at different stages were as follows: prescribing (47.8\%), transcribing (51.8\%), dispensing (33.6\%) and administration (70\%) (10). On the other hands, some of hospital managers declared that among 100 to 150 patients, one person dies from ME in hospitals, and the statistic of ME in 2012 was higher than that of 2011 (12\%) (11). Moreover, 24\% of complaints against physicians are about death related to MEs (12).

In general, in Iranian hospitals the highest frequency of MEs is related to systemic, administration and drug errors $(2,13)$. In addition, individual factors such as miscalculations of doses and inadequate knowledge of medications were the most frequent contributing factors in MEs among nurses (14).

Some studies in Iran showed that more of 50\% of MEs occurred in larger hospitals $(2,15)$. In addition, internal wards and morning shift, and also crowded ward are other influential factors in the incidence of MEs (2). Notably, nurses and physicians commit the most of errors in hospitals in Iran, respectively (67.3\% and 20.2\%) (4). According to the mentioned sentences, it is needed to be more sensitive about ME in healthcare organizations and prevention strategies must be used for decrease this challenge.

\section{Prevention Strategies}

The goal of error reduction and increased productivity and accuracy can be achieved through a system-focused approach that focuses on the process and has a systematic attitude. Top managers and staff should pay more attention to MEs reducing policies and strategies in health systems, particularly in hospitals. There are various strategies and methods to reduce MEs, which are as follows wristband bar-code medication scanning (1), computerized physician order entry (CPOE) (6), reporting medical error and near miss and use of root cause analysis method $(2,4)$; reporting of errors is crucial to the process of error management 
and is fundamental to the broad goal of error reduction. In addition, good policies and techniques, appropriate communications among medical staff, use of appropriate technologies (2), improvement of patient safety culture that rewards and learns from error reporting, and establishment of a feedback system to determine the factors contributing to error, are other strategies to prevent of errors in the health system. On the other hands, access to pharmacological text book, defining protocols, medication labeling and packages, patients' education about their treatment, physician education about appropriate guidelines for prescribing, providing information about new drugs, and compensation of staff shortage are prevention strategies in Iranian hospitals $(14,16)$. Using these simple strategies, managers and staff can prevent a high percentage of deaths and costs in hospitals (2). In general, authors recommended a reduction in staff's workload, compliance between the number of employees and workload, attention to crowded wards and morning shifts and improvement of error registration system to prevent MEs, especially in public hospitals.

\section{Footnote}

Authors' Contribution: All authors contributed in the writing of the manuscript and drafted the manuscript.

\section{References}

1. Khammarnia M, Kassani A, Eslahi M. The Efficacy of Patients' Wristband Bar-code on Prevention of Medical Errors: A Meta-analysis Study. Appl Clin Inform. 2015;6(4):716-27. doi: 10.4338/ACI-2015-06-R-0077. [PubMed: 26767066].

2. Khammarnia M, Ravangard R, Barfar E, Setoodehzadeh F. Medical errors and barriers to reporting in ten hospitals in southern iran. Malaysian J Med Sci. 2015;22(4):57.

3. Mortazavi SB, Oostakhan M, Mofidi A, Babaei A. The impact of patient safety climate on medical errors in a sample of nurses: Creating safer health care. Health Scope. 2013;1(4):158-64.
4. Khammarnia M, Ravangard R, Ghanbari Jahromi MAM. A study on the medical errors in public hospitals of shiraz, 2013. Hospital. 2014;13(3):17-24.

5. Dhatt GS, Damir HA, Matarelli S, Sankaranarayanan K, James DM. Patient safety: patient identification wristband errors. Clin Chem Lab Med. 2011;49(5):927-9. doi: 10.1515/CCLM.2011.129. [PubMed: 21288177].

6. Khammarni M, Sharifian R, Keshtkaran A, Zand F, Barati O, Khonia E, et al. Prescribing errors in two ICU wards in a large teaching hospital in Iran. Int JRisk SafMed. 2015;27(4):169-75. doi: 10.3233/JRS-150666. [PubMed: 26756890].

7. Waldman JD, Smith HL. Strategic planning to reduce medical errors: Part I-diagnosis. J Med Pract Manage. 2012;27(4):230-6. [PubMed: 22413600].

8. Tully MP, Ashcroft DM, Dornan T, Lewis PJ, Taylor D, Wass V. The causes of and factors associated with prescribing errors in hospital inpatients: a systematic review. Drug Saf. 2009;32(10):819-36. doi: 10.2165/11316560-000000000-00000. [PubMed:19722726].

9. Siddiqi S, Elasady R, Khorshid I, Fortune T, Leotsakos A, Letaief M, et al. Patient Safety Friendly Hospital Initiative: from evidence to action in seven developing country hospitals. Int J Qual Health Care. 2012;24(2):144-51. doi: 10.1093/intqhc/mzr090. [PubMed: 22302070].

10. Mansouri A, Ahmadvand A, Hadjibabaie M, Kargar M, Javadi M, Gholami K. Types and severity of medication errors in Iran; a review of the current literature. Daru. 2013;21(1):49. doi:10.1186/2008-2231-21-49. [PubMed: 23787134].

11. Barati O, Khammarnia M, Sadeghi A, Setoodehzadeh F. Prevention Approach of Medical Errors. Shiraz Univ Med Sci. 2015:1-248.

12. Siabani S, Alipour AA, Siabani H, Rezaei M, Daniali S. A survey of complaints against physicians reviewed at Kermanshah. J Kermanshah Univ Med Sci. 2009;13(1).

13. Mohsenzadeh A, Rezapour S, Birjandi M. Frequency of medical errors in hospitalized children in Khorramabad Madani hospital during six months in 2008. Yafteh. 2010;11(4):31-8.

14. Mansouri A, Ahmadvand A, Hadjibabaie M, Javadi M, Khoee SH, Dastan $\mathrm{F}$, et al. A review of medication errors in iran: sources, underreporting reasons and preventive measures. Iran JPharm Res. 2014;13(1):3-17. [PubMed: 24734052]

15. Pham JC, Story JL, Hicks RW, Shore AD, Morlock LL, Cheung DS, et al National study on the frequency, types, causes, and consequences of voluntarily reported emergency department medication errors. $J$ Emerg Med. 2011;40(5):485-92. doi: 10.1016/j.jemermed.2008.02.059. [PubMed: 18823735].

16. Dabaghzadeh F, Rashidian A, Torkamandi H, Alahyari S, Hanafi S, Farsaei S, et al. Medication errors in an emergency department in a large teaching hospital in tehran. Iran J Pharm Res. 2013;12(4):937-42. [PubMed: 24523775]. 